\title{
LA TÉCNICA DE PRECOLORACIÓN DE WALKER PARA EVALUAR Plasmodium vivax Grassi Y Plasmodium malariae Laveran EN COMUNIDADES ASHÁNINKAS EN SATIPO (JUNIN, PERÚ)
}

\author{
PRECOLORATION TECHNIQUE OF WALKER TO \\ EVALUATE Plasmodium vivax Grassi AND Plasmodium malariae Laveran IN \\ ASHANINKAS COMMUNITIES FROM SATIPO (JUNIN, PERU)
}

\author{
José A. Iannacone O.'; Cecilia Caballero R. \& José A. Rentería
}

\section{RESUMEN}

Se evaluó la técnica de precoloración de Walker en comparación con la técnica de Giemsa clásica como metodo de diferenciación para las especies de Plasmodium vivax y Plasmodium malariae, en 208 muestras de sangre de siete comunidades Ashäninkas en el distrito de Rio Tambo, Satipo, Junin, Perú. La densidad parasitaria por el sistema de cruces $(+)$ y por parásitos $/ \mathrm{mL}$ fue mayor en la técnica de precoloración de Walker. La prevalencia del parásito en el total de muestras evaluadas fue: $P$ vivax $(24,52 \%)$ y $P$ malariae $(0,48 \%)$. De las siete comunidades evaluadas en el distrito de Rio Tambo: Puerto Ocopa, Union Puerto Asháninka y Shimavenzo presentaron la mayor prevalencia de infección con $42,42 \%, 27,27 \%$ y $27,27 \%$ respectivamente. Las edades de 0 a 10 años y $41-50$ años presentaron la mayor prevalencia de infección con $31,30 \%$ y $40 \%$ respectivamente. La prevalencia de infección por plasmodio fue independiente del sexo. La densidad de parásitos $/ \mathrm{mL}$ por ambas lécnicas de diferenciación, fue mayor en la comunidad Puerto Prado, pero estadísticamente independiente de los grupos etáreos y el sexo. Se analizan las ventajas de la técnica de la precoloración de Walker y algunos factores que influirian en las variaciones en la prevalencia de infección y densidad parasitaria de los plasmodios en dependencia de las comunidades evaluadas, grupos etáreos y el sexo.

Palabras clave: Malaria, Plasmodium, diagnóstico parasitológico, comunidades nativas.

\begin{abstract}
The precoloration technique of Walker was assayed in comparison with the classic Giemsa technique as a differential method to Plasmodium vivax and Plasmodium malariae, in 208 blood samples of seven Ashaninka communities from Rio Tambo district, Satipo, Junin, Peru. Density of parasites by crossing system ( + ) and by parasites $/ \mathrm{mL}$ were higher with precoloration of Walker. The prevalence of the parasites was to $P$. vivax $(24,52 \%)$ and $P$. malariae $(0,48 \%)$ of all samples assayed. Of the seven communities evaluated from Rio Tambo district: Puerto Ocopa, Unión Puerto Ashaninka and Shimavenzo communities showed the highest prevalence of infection with $42,42 \%, 27,27 \%$ y $27,27 \%$ respectively. The ages between 0 to 10 and $41-50$ years showed the highest prevalence of infection with $31,30 \%$ and $40 \%$ respectively. The prevalence of infection was independent with the sex. Density of parasites $/ \mathrm{mL}$ with both lechniques were higher in Puerto Prado community; but statistically independent with age and sex. The advantage of precoloration technique of Walker was investigated and some factors that would influence variations in the prevalence of infection and density of parasites of plasmodia assayed dependent on what communities were sampled, age and sex.
\end{abstract}

Key words: Malarie, Plasmodium, parasitological diagnosis, native communities.

\section{INTRODUCCIÓN}

La malaria o paludismo es una enfermedad tropical causada por un protozoario del genero Plasmodium y transmitida por un mos-

1 Laboratorio de Ecofisiologia. Área de Biodiversidad Animal. Facultad de Ciencias Naturales y Matematicas. Universidad Nacional Federico Villarreal. Calle San Marcos 383, Pueblo Libre, Lima 21-Peru

E-mail: Iorena12@correo.dnet.com.pe quilo del género Anopheles, esta enfermedad es extremadamente peligrosa y se encuentra distribuida en climas tropicales alrededor del mundo (INS, 1996, Olliaro et al, 1996; Iannacone y Alvanino, 1997).

En el Perú, la malaria es una endemia nacional, con un patrón definido de comportamiento temporal y estacional, cuya mayor in- 
cidencia generalmente se registra durante los seis primeros meses del año, asociada geográfica y ecológicamente a zonas tropicales y desérticas irrigadas de la costa norte, selva montañosa nororiental, selva centro sudoriental y selva baja o llano amazónico oriental de nuestro país (MINSA, 1996).

En el Perú existen tres especies de Plasmodium capaces de infectar al hombre: Plasmodium vivax Grassi, Plasmodium falciparum Welch y Plasmodium malariae Laveran. La mayor parte de los casos registrados por el Ministerio de Salud (MINSA), corresponden a la infección por $P$, vivax, aunque hace algunos años se viene incrementando el número de casos de infección por $P$. falciparum, lo cual se asocia a una forma más crítica y grave de malaria (OMS, 1988; Demarini et al., 1995; MINSA, 1995). En la vertiente nororiental de los Andes y Llano amazónico (Loreto y Jaén), la especie predominante continúa siendo $P$. vivax, pero se observa gradualmente un aumento de malaria por $P$. falciparum y por $P$ malaricie (Chapilliquen et al., 1993; MINSA, 1996).

El examen microscópico de muestras sanguíneas empleando la coloración Giemsa clásica es un componente esencial para la vigilancia y control de la malaria en áreas rurales (Payne, 1988; Tham et al, 1999), En la última década, la investigación de esta enfermedad ha sido dirigida a la utilización de métodos para mejorar el diagnóstico de la malaria (WHO, 1988; Tharavanij, 1990; Shiff et al, 1993; Jelinek y Grobush, 1999; Kathleen y Zhong, 1999). Se han empleado muchas técnicas, entre ellas la microscopia fluorescente, técnicas moleculares ( «PCR»), prueba de captura rápida de antígenos con tiras reactivas (ParaSight@-F), y por último técnicas inmunoenzimáticas (Pammenter, 1988; Oliveira et al., 1996; Makler et al., 1998; Palmeretal, 1998). Sin embargo, en nuestro país, estos procedimientos aún no se emplean en evaluaciones de áreas endémicas, ni mucho menos en laboratorios de escasos recur- sos en zonas rurales tropicales. Una alternativa viable en nuestro país es el empleo de la técnica de precóloración de Walker, que modifica la coloración Giemsa clásica, mejorando la observación microscópica; es de bajo costo, y permite una lectura a posteriori sin pérdida de la forma y del color característico de los plasmodios, y además no requiere un adiestramiento muy especializado (OMS, 1988).

El presente estudio tuvo como objetivos: 1) evaluar la eficacia de la técnica de precoloración de Walker en comparación con el Giemsa clásico, como método de diferenciación para las especies de $P$. vivax y $P$. malariae registrados en el distrito de Río Tambo, Satipo (Junín, Perú), y 2) determinar la prevalencia de infección y densidad parasitaria de los plasmodios encontradas en el distrito de Río Tambo, Satipo en dependencia de las comunidades evaluadas, grupos etáreos y sexo.

\section{MATERIALES Y MÉTODOS}

\section{Situación geográfica del distrito Río Tambo}

El distrito Río Tambo, a $400 \mathrm{msnm}$, pertenece a la provincia de Satipo, departamento de Junín, región Mariscal Andrés Avelino Cáceres, Perú. La capital del distrito de Tambo es Puerto Ocopa. Este distrito tiene más de 20 comunidades entre nativos Asháninkas y colonos residentes de la zona; se localiza aguas abajo de la confluencia de los ríos Pangoa y Perené. Las siete comunidades seleccionadas para realizar el muestreo fueron Puerto Ocopa, Gloriabamba, Puerto Prado, Unión Puerto Ashaninka, Puerto Asháninka, Shimavenzo y Samaniato (Figura 1).

\section{Trabajo de Campo}

Número de muestras. El número de muestras tomadas fue en total 208. El muestreo de campo se realizó durante agosto y setiembre de 1997, distribuidas entre las siete comunida- 


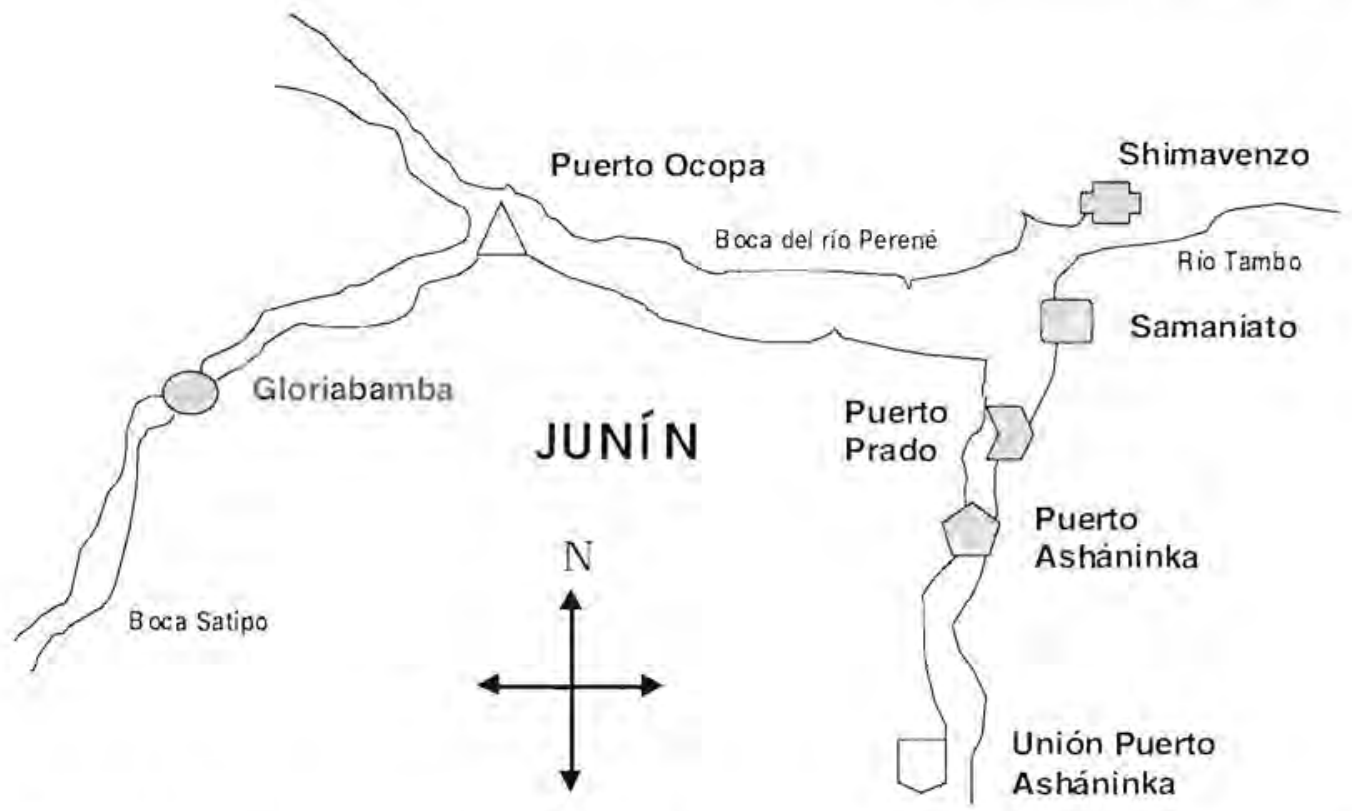

Figura 1. Mapa de las siete comunidades Asháninkas muestreadas en el distrito de Rio Tambo, Satipo, Junín. Perú.

des en orden doscendente al número de muestras: Unión Puerto Asháninka (66), Samaniato (26), Puerto Ocopa (33), Gloriabamba (22), Shimavenzo (22) Puerto Prado (20) y Puerto Asháninka (19).

Obtención de la muestra. La obtención de las muestras se realizó a través de la técnica de la gota gruesa, de acuerdo al procedimiento descrito por la OMS (1988). Se preparó dos clases de películas de sangre, la gota gruesa y el frotís pard su examen por microscopía directa, ambas en una misma lámina. De cada comunero se tomaron cuatro láminas, de las cuales dos fueron coloreadas con la técnica de precoluración de Walker al momento de la obtención de la muestra y las dos láminas restanter fueron coloreadas con la técnica Gremsa habitual de laboratorio, empleada por OMS (1988).

Técnica de precoloración de Walker. Esta técnica requiere del colorante azul de metileno $(0,3 \%)$ y de una solución amortiguadora (buffer fosfato). El portaobjetos se sumergió durante un segundo en azul de metileno, y se eliminó el exceso del colorante; la lámina se sumergió en la solución amortiguadora a través de cinco inmersiones suaves tratando de obtener una superficie clara no azulada, y por último se procedió al secado de la lámina a la temperatura ambiente.

Criterios de inclusión. Personas en estado febril, sexo masculino o femenino desde los tres meses de edad hasta los 50 años.

Criterios de exclusión. Persona que se encuentre en calidad de visitante en cada una de las siete comunidades muestreadas.

\section{Trabajo de Laboratorio}

En el laboratorio se fijaron los frotises con metanol, se colorearon las cuatro láminas, se diagnosticaron al microscopio las muestras hemáticas, y finalmente se procedió a determinar la densidad parasitaria a través de dos métodos: el sistema de cruces y el número de parásitos por microlitro de sangre (parásitos $/ \mu \mathrm{L}$ )

Diagnóstico de la muestra hemática. Se identificó la especie de plasmodio presente en cada lámina, se observaron los estadios característicos como trofozoito joven, mediano, 
maduro, esquizonte y gametocito. Se observaron las diferencias morfológicas puntuales entre $P$. vivax y $P$. malariae en base al tamaño del núcleo, forma del citoplasma, pigmento presente, número de merozoitos y tamaño del trofozoito maduro, etc., usando las claves de la OMS (1987), con estas características se determinó la especie del plasmodio presente en cada lámina.

\section{Determinación de la densidad parasi-} taria. A través de dos métodos:

Sistema de cruces (+). Se sumó el total de parásitos de todos los estadios observados con igual peso ponderado, en 100 campos microscópicos, siguiendo la siguiente escala:

$1 \quad \begin{aligned} & \text { Menos de } 40 \\ & 2 \quad+2\end{aligned} \quad \begin{aligned} & \text { De } 40 \text { a } 60 \text { parásitos en } 100 \\ & \text { campos. }\end{aligned}$
$3 \quad+\quad \begin{aligned} & \text { Un parásito por campo en } \\ & 100 \text { campos. }\end{aligned}$
$4 \quad+\quad \begin{aligned} & \text { De } 2 \text { a } 20 \text { parásitos por } \\ & \text { campo en } 100 \text { campos. }\end{aligned}$
$5 \quad+++\quad \begin{aligned} & \text { De } 21 \text { a } 200 \text { parásitos por } \\ & \text { campo en } 100 \text { campos. }\end{aligned}$
$6 \quad++++$
Más de 200 parásitos por
campo en 100 campos.

Número de parásitos por microlitro de sangre $(\mu \mathbf{L})$. Para realizar el conteo se necesitó de dos contadores manuales, uno para contar los estadios de los parásitos con igual peso ponderado y el otro para los leucocitos. Si después de contar 200 leucocitos, 10 o más parásitos han sido identificados, se deja de realizar el conteo y si después de contar 200 leucocitos se encontró 9 o menos parásitos se continúa contando hasta llegar a 500 leucocitos. El número relativo de parásitos con el número de leucocitos contados puede ser convertido a parásitos por microlitro $(\mu L)$ de sangre usando la siguiente fórmula:

Parásitos/ $\mu \mathrm{L}=\frac{\text { Número de parásitos }}{\text { Número de leucocitos }} \times 8000$

\section{Análisis de datos}

Se emplearon algunos métodos estadísticos de asociación: el coeficiente de correlación de Pearson ( $r$ ) se utilizó como indicación de la relación entre los grupos etáreos de las personas muestreadas y el número de parásitos/ $\mu \mathrm{L}$ con Giemsa clásico y con la precoloración de Walker. Se usó este coeficiente para determinar la relación entre grupos etáreos y la prevalencia de infección por plasmodios, con previa transformación arcoseno de los datos de prevalencia. El efecto del sexo en la prevalencia de infección se calculó usando la prueba de $x^{2}$. El estadístico $G$, se utilizó para determinar si existen diferencias en la prevalencia según grupos etáreos y comunidades muestreadas (Zar, 1996). Para evaluar si existen diferencias entre el número de parásitos/ $\mu \mathrm{L}$ de sangre entre el Giemsa clásico y la técnica de Walker, se usó la prueba de t de student para datos pareados. Se utilizó el ANDEVA, para determinar si existen diferencias significativas en la densidad parasita$\mathrm{ria} / \mathrm{mL}$ de sangre por ambas técnicas entre las siete comunidades del distrito de Río Tambo y según grupos etáreos; para el primer caso, las comunidades Puerto Asháninka y Samaniato se consideraron un solo grupo; y en el último caso, se consideraron solo tres grupos de edades: 0-10 años, 11-20 años y 2150 años, por el reducido número de casos positivos. En el caso de existir diferencias significativas se usó la prueba a posteriori de Tukey. Se calculó la prueba de t de student para determinar si existen diferencias en la densidad parasitaria/ $\mu \mathrm{L}$ según el sexo de las personas muestreadas previa evaluación de la homogeneidad de varianzas empleando la prueba de Levene. El nivel de significancia fue evaluado a $\alpha=0,05$ (Daniel, 1993). $\mathrm{La}$ terminología ecológica para la prevalencia y densidad parasitaria de infección siguió los criterios de Bush et al. (1997). Para el cálculo de los parámetros estadísticos descriptivos e inferenciales, se empleó el paquete estadístico SPSS para Windows 95. 


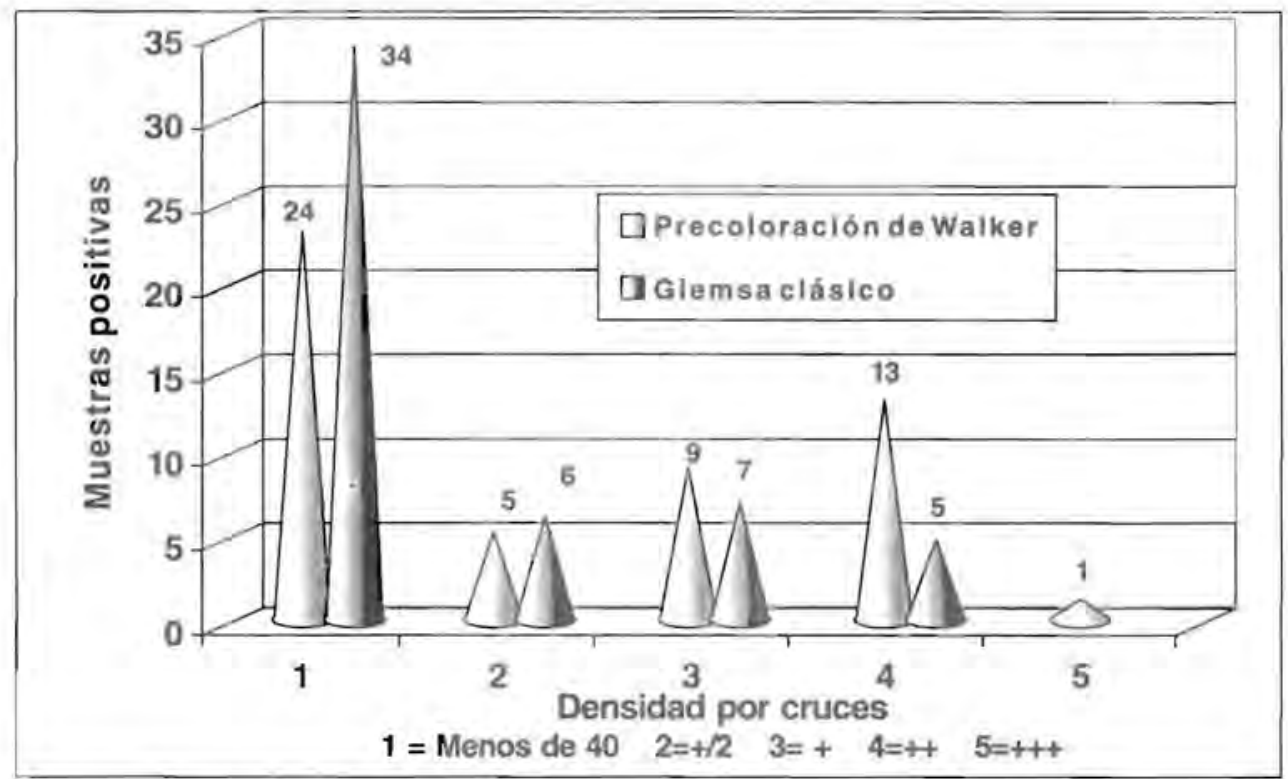

Figura 2. Densidad parasitaria de Plasmodiumspp. con la precoloración de Walker y con la coloración Giemsa clásica usando el sistema de cruces en comunidades Asháninkas de Satipo, Junin, Perú.

\section{RESULTADOS}

De las 208 muestras evaluadas se encontraron 52 muestras positivas a plasmodios, de los cuales 51 muestras $(98,08 \%)$ correspondieron a $P$. vivax y una muestra corresponde a P. malariae (1,92\%), lo que indica que la presencia de $P$. malariae es escasa en comparación con $P$. vivax. La prevalencia de las especies diagnosticadas en el distrito de Río Tambo para $P$. vivax es $24,52 \%$ y para $P$. malariae es $0,48 \%$ del total de muestras evaIuadas. El resto de casos febriles correspondería a otros agentes etiológicos distintos de la malaria.

Al comparar la densidad parasitaria hallada a través del sistema de cruces $(+)$ de las muestras tratadas con la precoloración de Walker y con la coloración Giemsa clásica, se observó en las muestras con la precoloración una mayor densidad parasitaria, incrementando notablemente en la escala 4 en un $160 \%$ y por ende, se reduce el número de muestras de baja densidad en un $29,41 \%$. En las muestras con Giemsa clásico, se observó un patrón opuesto (Figura 2).
La densidad parasitaria hallada a través del cálculo del número de parásitos por $\mathrm{mL}$ de sangre mostró una mayor cantidad de parásitos en láminas tratadas con la precoloración de Walker (2043,53 $\pm 4631,45)$, comparada con láminas coloreadas solo con el Giemsa clásico $(1620,11 \pm 3926,26)$ donde el número de parásitos encontrados fue menor $(t=2,88$, $\mathrm{P}=0,006, \mathrm{~g} .1 .=51$ ) (Figura 3). Además, ambos procedimientos de coloración están altamente correlacionados $(\mathbf{r}=0,98, \mathrm{P}=0,001$, $\mathrm{n}=52$ ). La frecuencia de dominancia para la densidad de parásitos/ $\mu \mathrm{L}$, de las dos técnicas empleadas, en Giemsa clásico fue superior en nueve casos $(17,30 \%)$, la técnica de precoloración de Walker en 38 casos $(73,07 \%)$ y una frecuencia compartida en cinco casos $(9,61 \%)$.

La figura 4 describe el numero de casos de malaria positivos y negativos en las siete comunidades del distrito de Río Tambo, Satipo, Junín. El número de muestras positivas en las siete comunidades presentó el siguiente orden decreciente: Unión Puerto Asháninka > Puerto Ocopa > Shimavenzo > Gloriabamba > 


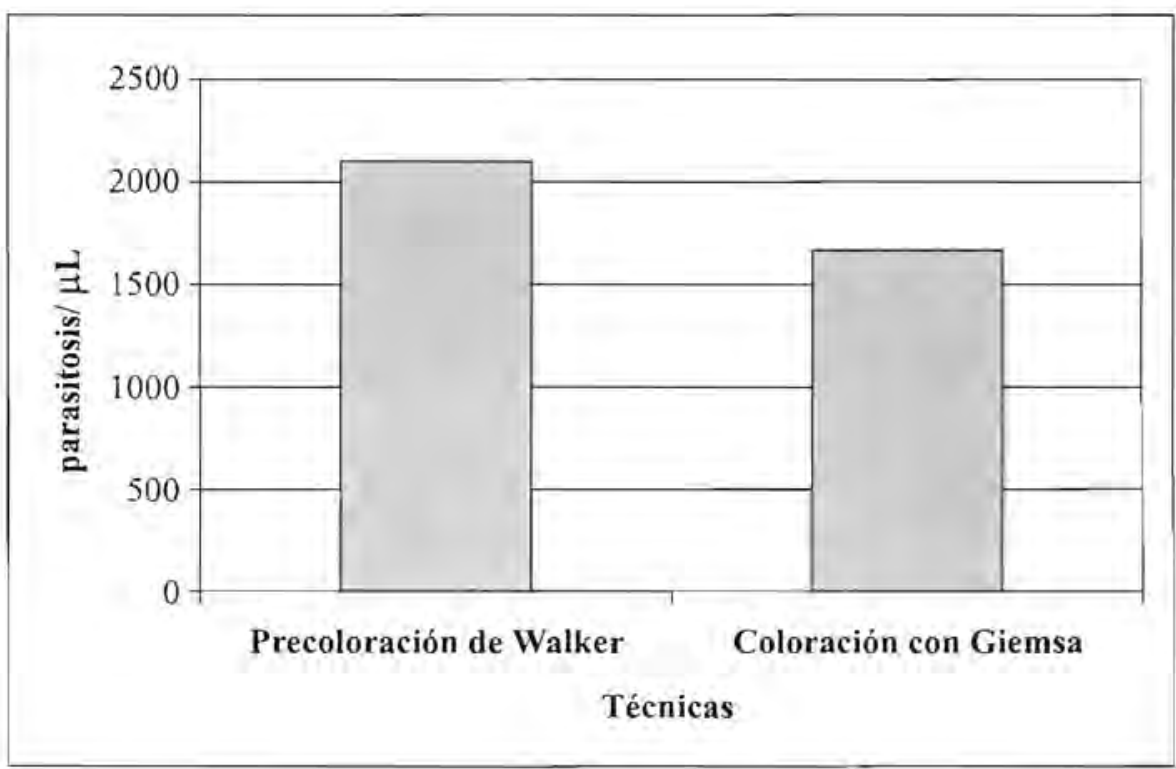

Figura 3. Densidad parasitaria de Plasmodium spp. con la precoloración de Walker y la coloración Giemsa clásica usando el sistema de parásitos/ $\mu \mathrm{L}$ en comunidades Asháninkas de Satipo, Junín, Perú.

Samaniato $=$ Puerto Prado $>$ Puerto Asháninka. Unión Puerto Asháninka y Puerto Ocopa son las dos comunidades que presentaron la mayor población en comparación con el resto de comunidades visitadas. Existen diferencias en la prevalencia de infección entre las siete comunidades de Río Tambo $(\mathrm{G}=12,06, \mathrm{P}<0,05, \mathrm{~g}, \mathrm{I}=6)$. La prevalencia de los casos de malaria en las siete comunidades de Río Tambo presentó el siguiente orden decreciente: Puerto Ocopa $(42,42 \%)>$ Unión Puerto Asháninka $(27,27 \%)=$ Shimavenzo $(27,27 \%)>$ Gloriabamba $(22,72 \%)>$ Puerto Prado $(20 \%)>$ Samaniato $(15,38 \%)>$ Puerto Asháninka $(5,26 \%)$. El ANDEVA indica que existen diferencias significativas en la densidad parasitaria/ $\mu \mathrm{L}$ de sangre para las siete comunidades, por la precoloración $(\mathrm{F}=4,27, \mathrm{P}=0,003$, g.l. $=5$ ) y por el Giemsa clásico $(F=3,61, P=0,008$, g.l $1=5)$. Para ambas técnicas de coloración, la prueba de Tukey indicó que Puerto Prado fue diferente del resto de comunidades en relación al número de parásitos $/ \mathrm{mL}$ (Precoloración $=10758$ y Giemsa clásico $=8528$ ).
Se observa que de los 52 casos positivos para malaria encontrados, de acuerdo a la edad, 47 casos $(88,46 \%)$ corresponden a niños y jóvenes entre 0 a 20 años de edad el análisis estadístico muestra que la presencia de malaria es dependiente de la edad $(\mathrm{G}=13,97, \mathrm{P}<0,05$, g.l $=4)$, lo que nos revela que los niños de 0 a 10 años (Prevalencia $=31,30 \%)$ y los adultos de 41 a 50 años (Prevalencia $=40 \%)$, representan la población más susceptible a contraer esta enfermedad (Figura 5). Sin embargo, la edad de la población muestreada no se encuentra correlacionada con la prevalencia de infección por plasmodio $(r=0,05, P=0,937, n=5)$. Sin embargo el ANDEVA indica que el número de parásitos por $\mathrm{mL}$ de sangre es estadísticamente igual tanto por la precoloración $(\mathrm{F}=1,24, \mathrm{P}=0,29, \mathrm{~g} .1 . \mathrm{l}=2 \mathrm{y}$ 49) como por el Giemsa clásico ( $F=1,09$, $\mathrm{P}=0,34, \mathrm{~g} . \mathrm{I}=2$ y 49 ) con relación a los tres grupos etáreos evaluados, aunque numéricamente el grupo etáreo de 0 a 10 años fue mucho mayor que los otros dos grupos, según ambas técnicas (Pre 


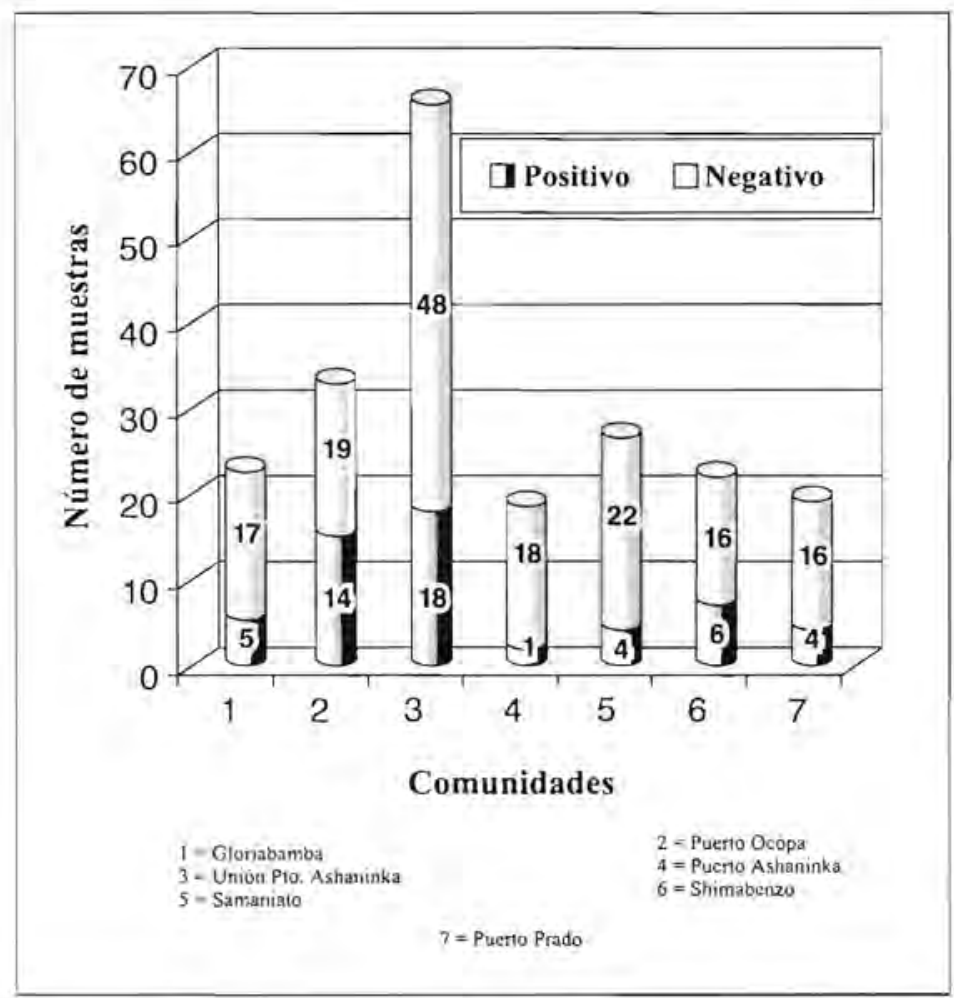

Figura 4. Número de muestras positivas de Plasmodium spp, en siete comunidades Asháninkas de Satipo, Junin. Perú

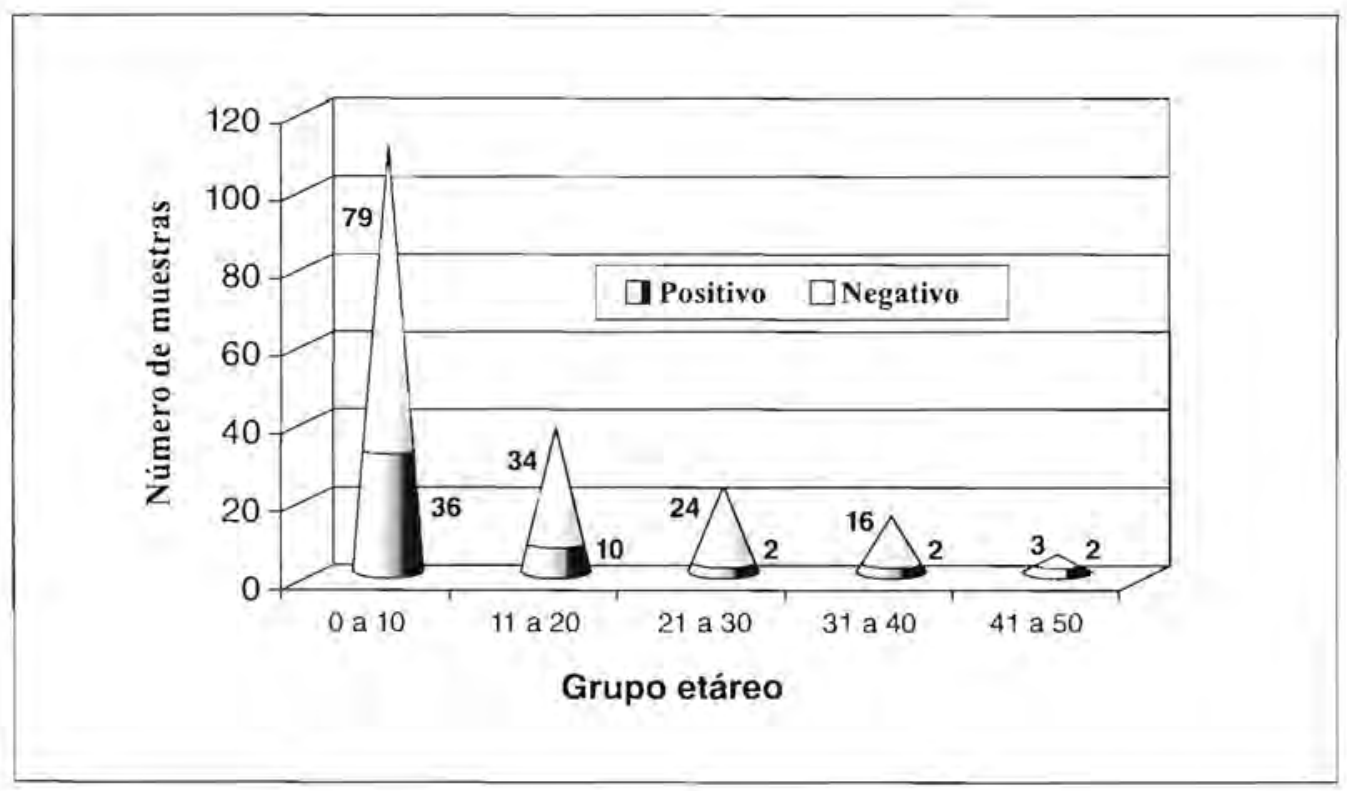

Figura 5. Número de muestras con Plasmodiumspp. de acuerdo a los grupos etáreos en las comunidades Asháninkas de Satipo, Junín, Perú. 
coloración $=2710,27 \pm 5430)$ y (Giemsa clási$\mathrm{co}=2148,52 \pm 4613$ ) (Figura 6).

La presencia de los casos positivos de malaria en relación al sexo de los Asháninkas muestra independencia en las comunidades del distrito de Río Tambo $\left(\mathrm{x}^{2}=0,025, \mathrm{P}>0.05\right.$. g.l. = 1). Además, la prueba de $t$ de student indica que no existen diferencias en la densidad parasitaria/ $\mu \mathrm{L}$ según el sexo, por la precoloración $(\mathrm{t}=1,59, \mathrm{P}=0,1 \mathrm{l}, \mathrm{g} .1 .=50) \mathrm{y}$ por el Giemsa clásico $(t=1,81, \mathrm{P}=0,07$. g.I. $=50$ ).

\section{DISCUSIÓN}

Durante 1996, se presentaron solo tres casos de $P$. malariae en el departamento de Junín, lo que representa una prevalencia anual del $0,002 \%$ del total de casos evaluados (INS, 1997); sin embargo, este porcentaje podría incrementarse, pues en muchas ocasiones algunos técnicos microscopistas lo confunden con $P$. vivax, debido a la falta de entrenamiento y experiencia. El resultado obtenido en este estudio; es una orden de magnitud menor a la prevalencia para $P$. malariae de $0,06 \%$ para el Perú durante el año 1996. Bambaren el al. (1997) indican que durante 1996, en el distrito de Perené, Junín, el 100\% de casos fue por $P$. vivax y que el departamento de Junín corresponde a una zona de alto riesgo para malaria. El estudio se realizó en época considerada de baja transmisión de malaria, lo cual reforzaría la hipótesis de las presencia de recaidas en algunas comunidades, en especial en Puerto Prado, el cual es un fuerte militar.

La mayor densidad parasitaria nor el sistema de cruces $(+)$ y por el número de parásitos/mL de sangre, en las muestras tratadas con la precoloración de Walker en comparación con el Giemsa clásico (Figura 2 y 3 ), se explica porque la precoloración logró una óptima deshemoglobinización evidente con un fondo blanco y claro, y esto nos permitió diferenciar la especie de plasmodio presente en la lámina, al observar los colores y formas características del parásito en láminas de baja densidad, mejorando la observación microscópica de las láminas coloreadas en comparación con la técnica de Giemsa clásica, lo que disminuye los errores en el diagnóstico, ya sea por la identidad de la especie o por láminas de baja densidad, sobre todo en laboratorios locales de escasos recursos materiales y con pocas posibilidades de acondicionamiento para realizar otras técnicas como la inmunofluorescencia, las moleculares, etc. Además evita la fijación de la muestra, destinada sobre todo para zonas rurales donde el transporte al laboratorio local toma tiempo, como es el caso del distrito de Río Tambo. En Satipo (Junín), se presentan dos especies de plasmodio como son $P$. vivax y $P$. malariae; la identidad de ambas especies conlleva a mucha confusión y error de lectura, dado que ambos parásitos presentan una morfología muy parecida, no se diferencian ambas especies, sobre todo si el microscopista no es experto y si tiene por limitaciones de un microscopio de no muy buena resolución; esto tiene como consecuencia un tratamiento ineficaz.

Las diferencias encontradas en la prevalencia de infección por plasmodio, según las siete comunidades muestreadas, fue mayor en Puerto Ocopa, Unión Puerto Asháninka y Shimabenzo; estos resultados se deben según Kroeger y Alarcón (1993) a ligeras diferencias con relación a la cercanía a criaderos de culícidos. En nuestro trabajo las encuestas most iaron que miembros de una misma familia estaban infectados, debido aparentemente a que los vectores vuelven a las mismas viviendas y el mismo vector pica a diferentes miembros de una misma familia. Por otro lado, la población no usa mosquiteros, lo que incrementaría la posibilidad de contacto con el vector intradomiciliariamente, a pesar de no ser época de lluvias y, por lo tanto, se esperaría una menor densidad poblacional vectorial; sin embargo, la prevalencia de infección por los plasmodios en general es alta, inclusive si tomamos en cuenta que la población en cada una de las siete comunidades no sobrepasa los 300 habitantes. Además, una de las mayo- 


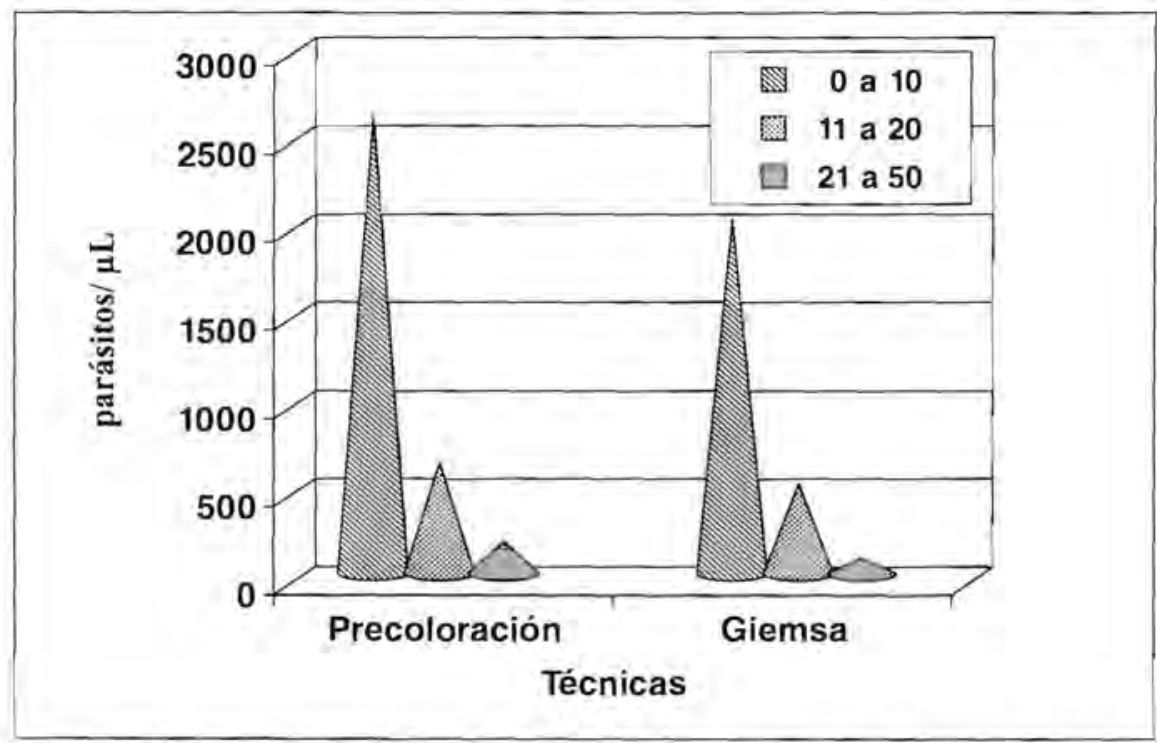

Figura 6. Comparación de la densidad parasitaria de Plasmodium $\$ p p . / \mu \mathrm{L}$ por grupos etáreos en las comunidades Asháninkas de Satipo, Junin, Perú con la precołoración de Walker y con la coloración Giemsa.

res diferencias en la prevalencia de infección entre las siete comunidades sería explicada por las variaciones en los grupos etáreos y de sexo en las siete comunidades. Puerto Prado presentó un mayor número de parásitos $/ \mathrm{mL}$ en comparación con las otras seis comunidades, debido a que toda la población evaluada correspondió a soldados jóvenes, a quienes recién se les permitió realizarse un análisis de diagnóstico, al presentar fiebres periódicas y con una fase muy avanzada de la enfermedad. El grado de accesibilidad a las siete comunidades que se encuentran en un proceso de repoblamiento, debido a los efectos del terrorismo, no influyó marcadamenté en los resultados obtenidos, pues Puerto Ocopa que es la más accesible y que presenta puesto de salud y Unión Puerto Asháninka, que es la menos accesible, no presentaron en ningún caso diferencias significativas.

En general, las comunidades por grupos etáreos siguieron uno de los tres patrones de comportamiento, es decir, niveles descendentes de prevalencia de infección con la edad, como sucede con un estudio realizado en La Cam- piña, Alto Piura, Perú, cuyos valores mayores suben entre 0-4 años y a 54 años de edad (Kroeger y Alarcón, 1993). Esto nos indicaria una mayor proporción de infección intradomiciliaria. Bambaren el al. (1997) en el distrito de Perené, Junin, Perú, indican que el grupo etáreo más afectado correspondió a la población entre 15 a 44 años, atribuible a una mayor infección extradomiciliaria. Nuestros resultados fueron opuestos a lo encontrado por estos autores.

El que no existan diferencias en la prevalenciay densidad parasitarial $\mu \mathrm{L}$ de sangre con el sexo de los individuos muestreados se explicaria porque no habria hábitos marcados entre hombres y mujeres que originaran grupos con exposición al vector (Kroeger y Alarcón, 1993; Castilló y Silva, 1997). Sin embargo, algunos estudios realizados, también en selva baja como Madre de Dios, indican una mayor prevalencia de infección en el sexo mas culino (Kroeger y Alarcón, 1993).

Agradecimientos: A la Blga. Lorena Alvariño F, del Laboratorio de Ecofisiologia de la Universidad Nacional Federico Villarreal, 
Perú, por la revisión crítica del presente manuscrito. A la Blga. Cecilia Arce B. y a la Lic. en Tec. Med. Blanca Pardavé L. de la División de Parasitología del Instituto Nacional de Salud, Perú, por las sugerencias y apoyo brindado a la presente investigación. A los técnicos José Reátegui L. y Deysi Valenzuela M. del Centro de Salud de Puerto Ocopa, Satipo, Junín, Perú por su colaboración en el trabajo de campo con las comunidades Asháninkas.

\section{LITERATURA CITADA}

Bush, A. O.; K. D. Lafferty; J. M. Lotz y A. W. Shostak. 1997. Parasitology meets ecology on its own terms: Margolis et al revisited. J. Parasitol. 83: 575-583,

Bambaren, C.; J. Laos; C. Rebaza y R. Azareño, 1997. Algunos aspectos epidemiológicos de los casos reportados de malaria en el distrito de Perené, Junín, 1996. Bol. Per. Parasitol, 12:61.

Castillo, A y T. Silva. 1997. Malaria en Niños menores de seis años de las comunidades nativas y caserios de colonos del alto Marañón, Imaza -Prov. Bagua Bol. Per, Parasitol. 12: 63.

Chapilliquén, F. C. Arce y B. Pardavé. 1993. El laboratorio referencial de malaria y su evolución en el programa nacional de Malaria-MINSA 19801992. XI Congreso Latinoamericano de Parasitología. 21 al 26 de noviembre, Libro de Resúmenes. p. 31.

Daniel, W. W. 1993. Bioestadística: Base para el análisis de las ciencias de la salud. 3. Edición. Limusa. México. D.F., 667 pp.

Demarini, C. J.; R. Carhuallanqui y J. M. Demarini. 1995. Malaria en la Selva Central del Perú. II Congreso Peruano de Parasitología. 1-5 Julio. Libro de Resúmenes, p, 20.

lannacone, J. O. y L. Alvariño, 1997. Peces larvívoros con potencial para el control biológico de estados inmaduros de zancudos en el Perú. Rev. per. Ent. 40: 9-19.

INS (Instituto Nacional de Salud). 1996. Manual de laboratorio de malaria. Ministerio de Salud (MINSA), $35 \mathrm{pp}$

INS (Instituto Nacional de Salud), 1997. Informe de supervisión a los laboratorios intermedios y locales en la subregión de salud Junín realizado del 15 at 20 de diciembre de 1996. Copia mimeografiada. 25 pp.

Jelinek, T. y M. P. Grobush, 1999. Sensitivity and specifity of dipstick lest for rapid diagnosis of Malaria in nonimmune travelers. J. Clin. Microbiol, 37: 721-723.
Kathleen, J y Y. Zhong. 1999. Evaluation of a colorimetric PCR-bassed assay to diagnose Plasmodium falciparum Malaria in travelers. J. Clin. Microbiol. 37: 339-341

Kroeger, A. y J. Alarcón. 1993. Malaria en Ecuador y Perú y estrategias alternativas de control. Editorial Abya-Yala. Lima, Perú. 316 pp.

Makler, M., C. Palmer y A. Ager, 1998, A review of practical techniques for the diagnosis of malaria. Ann. Trop. Med. Parasitol 92: $419-433$

MINSA (Ministerio de Salud), 1995. Manual de procedimientos técnicos para el diagnostico de malaria. Serie de normas técnicas $N{ }^{\circ}$ 14: 1-25.

MINSA (Ministerio de Salud). 1996. Programa de control de malaria y otras enfermedades metaxénicas. situación epidemiológica de la malaria en el Perú. Copia mimeografiada. 42 pp.

Oliveira, D.A.; Y.P. Shi; A.J. Oloo; D.A. Boriga; B.L. Nahlen; W.A. Hawley; B.P. Holloway y A.A. Lai. 1996. Field evaluation of polymerase chain reaction-based nonisotopic liquid hybridization assay for malaria diagnosis. J. Infect Dis. 173 1284-1287.

Olliaro, P; J. Cattani y D. Wirth. 1996. Malaria, the submerged disease. JAMA 275: 230-233.

OMS (Organización Mundial de la Salud). 1987. La biología de los parásitos del paludismo. Serie de informes técnicos 743:1-12.

OMS (Organización Mundial de la Salud). I988. Diagnostico de la malaria. Series de informes técni$\cos$ 744:39-86.

Pălmer, C; J, Lindo; W Klaskala; J. Quesada; R. Kaminski; M. Baum y A. Ager. 1998. Evaluation of the optimal test for rapid diagnosis of Plasmodium vivax and Plasmodium falciparum. J. Clin. Microbiol. 36:203-206.

Pammeter, M. 1988. Techniques for the diagnosis of malaria. S. Atr. med J. 74;55-57.

Payne, D. 1988. Use and limitations of light microscopy for diagnosing malania at the primary health care level. Bull. World Health Organ. 66: 621-626.

Shiff, C.J,; Z. Premi y J.N. Minjas, 1993. The rapid manual ParaSight(10-F test, A new diagnostic tool for Plasmodium falciparum infection. Trans Roy, Soc. Trop. Med Hyg 87: 616-618.

Tham, J. M.; S. H. Lee; T. M. C. Tan y C. Robert. 1999. Detection and species determunation of Malaria parasites by PCR: Comparison with microscopy and with Parasightk - F and ICT Malariae Pf tests in a clinical environment. J. Clin. Microbiol. 37: 1269-1273.

Tharavanij, S. 1990. New developments in malaria diagnosis techniques. Southeast Asian J. Trop. Med. Public Health 21:3-16.

WHO (World Health Organization), 1988, Malaria diagnosis: Memorandum from a WHO Meeting. Bull. World Health Org, 66: 118-125.

Zar, J. H. 1996. Biostatistical Analysis. Er. Ed Prentice-Hall, Inc. Upper Saddle River. New Yersey. 662 pp. 